\title{
PRIMES AND ANNIHILATORS
}

\author{
BY STEPHEN MCADAM
}

Communicated by I. N. Herstein, August 4, 1969

It is well known that in a commutative Noetherian ring, a prime ideal minimal over the annihilator of a finitely generated module is the annihilator of some element of that module. A short proof is given here. We use a lemma which strengthens an observation of Herstein by restricting attention to annihilators within a fixed prime ideal.

Lemma. Let $R$ be a commutative ring, $P$ a prime ideal, and $A$ an $R$-module. Suppose $a \in A$ with $I=\operatorname{Ann}(a) \subset P$. If $I$ is maximal with respect to being an annihilator of an element of $A$ and being contained in $P$, then $I$ is prime.

Proof. Suppose $x y \in I$, but $x \notin I$ and $y \notin I$. Then $I$ is properly contained in $(I, y)$ and $(I, y) \subset \operatorname{Ann}(x a)$. The maximality of $I$ implies $\operatorname{Ann}(x a) \nsubseteq P$. Pick $s \notin P, s \in \operatorname{Ann}(x a)$. We have $I \subset \operatorname{Ann}(s a)$ and $x \in \operatorname{Ann}(s a)$ so that $(I, x) \subset \operatorname{Ann}(s a)$. But $I$ is properly contained in $(I, x)$. Again by the maximality of $I$, Ann $(s a) \nsubseteq P$. Pick $t \notin P$, $t \in \operatorname{Ann}(s a)$. Thus $t s a=0$ and $t s \in \operatorname{Ann}(a) \subset P$. This is impossible since $s \notin P$ and $t \notin P$.

THEOREM. Let $R$ be a commutative Notherian ring, and $A$ a finitely generated $R$-module. If $P$ is a prime ideal of $R$ minimal over $\operatorname{Ann}(A)$, then there is an $a \in A$ with $P=\operatorname{Ann}(a)$.

Proof. Say $A$ is generated by $a_{1}, \cdots, a_{n}$. Then $\operatorname{Ann}(A)$ equals the intersection, for $i=1, \cdots, n$, of $\operatorname{Ann}\left(a_{i}\right)$. Since $\operatorname{Ann}(A) \subset P$, we have for some $i, \operatorname{Ann}\left(a_{i}\right) \subset P$. Because $R$ is Noetherian and $P$ contains the annihilator of some element of $A$, we can find an ideal $I \subset P$ such that $I$ is maximal with respect to being an annihilator of an element of $A$ and being contained in $P$. Since $I$ is the annihilator of some element of $A$, we have $\operatorname{Ann}(A) \subset I$. Thus $\operatorname{Ann}(A) \subset I \subset P$. By the lemma, $I$ is prime. Since $P$ is minimal over $\operatorname{Ann}(A)$, we must have $P=I$. Therefore $P$ is the annihilator of some element of $A$.

The reader will note that we did not use the full strength of $R$ being Noetherian, but only that it satisfied the ascending chain condition on annihilators of elements of $A$.

AMS Subject Classifications. Primary 1645; Secondary 1610.

Key Words and Phrases. Primes minimal over an annihilator, Noetherian ring.

University of Chicago, Chicago, Illinois 60637 